\title{
ERGONOMIA DA SALA DE AULA PARA ALUNOS COM TDAH: UMA REVISÃO SISTEMÁTICA
}

\author{
MORAIS, Maria Juliana C. de (1); \\ PEDROSA, Raphalea V. F. (2) \\ VILLAROUCO, Vilma (3) \\ (1) UFPE, Pós Graduação \\ e-mail:ju morais86@hotmail.com \\ (2) Esuda, Graduação \\ e-mail:raphaela.veloso@outlook.com \\ (3) UFPE, Dra. Eng. \\ e-mail:vvillarouco@gmail.com
}

\begin{abstract}
RESUMO
Muito é estudado sobre Transtorno de Déficit Atenção e Hiperatividade (TDAH) no âmbito da medicina, psicologia e pedagogia, bem como acontece com a sala de aula, na área pedagógica. Este artigo objetiva trazer a questão para o foco do ambiente construído, através de uma revisão sistemática de literatura (RSL), sobre como a ergonomia da sala de aula pode influenciar 0 aprendizado de alunos com TDAH. Com a RSL foi possível observar que existem poucas publicações, nos motores de busca utilizados, sobre o tema. Entretanto, nos os artigos selecionados, foi possível coletar informações e gerar um direcionamento para estudos futuros sobre o assunto.
\end{abstract}

Palavras chave : RSL; TDAH; ergonomia e sala de aula.

\begin{abstract}
Much is studied about Attention Deficit Disorder with Hyperactivity (ADDH) in medicine field, psychology and pedagogy, as well as with the classroom, with a pedagogical view. This article aims put the problem under built environment contexto through a Systematic Literature Review in order to analyze the most recente and relevant publications on how classroom's ergonomics environment can influence the learning process of students with ADDH.The SLR shows that there are few studies published in the search websites used, Capes and Scielo about the subject. However, with those selected articles, it was possible to collect information that point a direction for further studies on this topic.
\end{abstract}

Keywords: SLR; ADDH; Ergonomics environment and classroom.

\section{INTRODUÇÃO}

Buscando explorar bibliografias sobre o tema, esta revisão sistemática encontrou um elo ainda em formação entre abordagens na ergonomia do ambiente construído da sala de aula, ao tema TDAH, com poucas referências temáticas. A partir desta revisão, é possível 


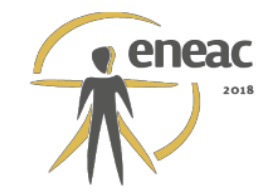

entender o teor e técnica de pesquisa utilizada para encontrar poucos resultados relacionados com o tema. Sendo possível encaixar em filtros os temas centrais e palavras chave para a pesquisa do assunto.

As pessoas com o Transtorno de Déficit de Atenção e Hiperatividade demonstram um padrão de agitação que as impossibilitam de fixar a atenção em estímulos importantes, prejudicando suas capacidades cognitivas e gerando um atraso no desenvolvimento, bem como nas habilidades reflexivas. A partir desse dado, conclui-se que a sala de aula é um dos locais que a criança passa a maior parte do dia, e é na sala que estímulos importantes são incentivados a fim de promover um desenvolvimento intelectual e social destas crianças.

Visto isso, o objetivo desta revisão sistemática da literatura é formar um referencial teórico a partir de artigos publicados, de como o ambiente da sala de aula pode auxiliar no desenvolvimento e aprendizagem de um aluno com TDAH.

\section{METODOLOGIA}

De acordo com Atallah e Castro (1997) a Revisão Sistemática de Literatura (RSL) é um método de meta nálise que torna os resultados de uma pesquisa ainda mais precisos, por se tratar de dados quantitativos e sem inconsistência de informações. Algumas de suas vantagens são descobrir as lacunas ainda existentes na temática pesquisada, além de ajudar a direcionar novas pesquisas.

A pesquisa limitou-se às seguintes plataformas de busca escolhidas foram:

- SCIELO: Library Online, iniciou desde 1997 e desde 2002 conta com o apoio da CNPq. Conta com 1249 periódicos e 573.525 artigos.

- Periódicos Capes: A Coordenação de Aperfeiçoamento de Pessoal de Nível Superior criou esta plataforma de acesso a importantes periódicos de divulgação científica e desde 1990 busca fortalecer a pós-graduação, oferecendo acesso a textos completos de artigos selecionados de mais de 21.500 revistas nacionais e internacionais.

O primeiro passo da investigação foi a definição de palavras-chave a partir do título e objeto do estudo, que a princípio foram, ergonomia, sala de aula e TDAH. Essas palavras foram pesquisadas, através de combinações entre elas, no dia 12 de abril de 2017, na plataforma de pesquisa Capes (http://www.periodicos.capes.gov.br), não obtendo nenhum resultado nas buscas. Conclui-se, portanto, que no âmbito da limitação do idioma português, o tema em questão ainda não possui trabalhos divulgados em periódicos catalogados nesse portal, podendo ser um tema ainda recente ou por existir uma lacuna nas linhas de pesquisa sobre tal assunto.

Uma vez que não foram encontrados artigos relacionados diretamente com o tema, fez-se necessário a ampliação do número de palavras-chave, e consequentemente de combinações entre elas, principalmente no âmbito da ergonomia do ambiente construído e da percepção ambiental. As palavras selecionadas foram listadas na tabela 1, junto com suas correspondentes na língua inglesa.

Tabela 1: Palavras-chave e suas correlações na língua inglesa.

\begin{tabular}{|l|l|}
\hline \multicolumn{2}{|c|}{ PALAVRAS-CHAVE } \\
\hline Português & Inglês \\
\hline
\end{tabular}




\begin{tabular}{|c|c|}
\hline Ergonomia do ambiente construído & $\begin{array}{c}\text { Build environment ergonomics or indor } \\
\text { ergonomic environment }\end{array}$ \\
\hline Percepção ambiental & Environmental perception \\
\hline Sala de aula & Classroom \\
\hline TDAH & ADHD \\
\hline Ambiente de estudo & Place of study \\
\hline Deficiência cognitiva & Cognitive deficiency \\
\hline
\end{tabular}

Fonte: As autoras, 2017.

As novas pesquisas foram feitas no dia 18 de abril e 16 de maio de 2017 no portal da Capes e no dia 23 de maio de 2017 no portal da Scielo (http://www.scielo.org/php/index.php). De acordo com as strings de busca encolhidas por junção das palavras chaves em pares, conforme mostra a tabela 2, e aplicada no campo de busca das plataformas (CAPES E SCIELO), foi permitido colher os primeiros números e o denominar os "resultados da busca", demonstrado nas próximas tabelas.

Tabela 2: Combinações entre as palavras-chave

\begin{tabular}{|l|l|}
\hline \multicolumn{2}{|c|}{ COMBINAÇÕES ENTRE PALAVRAS CHAVES } \\
\hline $\mathbf{1}$ & Ergonomia do ambiente + sala de aula \\
\hline $\mathbf{2}$ & Percepção ambiental +TDAH \\
\hline $\mathbf{3}$ & sala de aula + TDAH \\
\hline $\mathbf{4}$ & Percepção ambiental + sala de aula \\
\hline $\mathbf{5}$ & Ambiente de estudo + deficiência cognitiva \\
\hline $\mathbf{6}$ & Sala de aula + deficiência cognitiva \\
\hline $\mathbf{7}$ & Ergonomia do ambiente + ambiente de estudo \\
\hline $\mathbf{8}$ & Ambiente de estudo + TDAH \\
\hline
\end{tabular}

Fonte: As autoras, 2017.

Nas combinações com uma quantidade de resultados de busca maior que 30 , foram aplicados filtros em etapas. Primeiramente excluímos os artigos pela sua data de publicação, deixando os publicados apenas nos últimos 5 anos. Em seguida, a exclusão se deu após o filtro de revisão por pares e idiomas (apenas inglês e português), e por fim, após a leitura dos títulos dos artigos. Se fizesse necessário uma quarta e última etapa da triagem, essa seria realizada através da leitura do resumo. Sendo selecionados apenas os que obtiveram um conteúdo satisfatório para contribuir com o objetivo da pesquisa. 


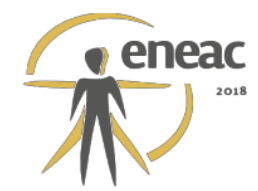

\section{RESULTADOS}

Realizado todo o procedimento explanado na metodologia, a tabela 3 retrata os primeiros resultados obtidos através da plataforma Capes, com as pesquisa realizada em português.

Tabela 3: Quantitativo de resultados encontrados pelas combinações em português na CAPES

\begin{tabular}{|c|c|c|c|}
\hline CAPES & COMBINAÇÕES & $\begin{array}{c}\text { RESULTADOS } \\
\text { DA BUSCA }\end{array}$ & $\begin{array}{c}\text { RESULTADOS } \\
\text { RELEVANTES }\end{array}$ \\
\hline $\mathbf{1}$ & Ergonomia do ambiente + Sala de aula & 17 & 1 \\
\hline $\mathbf{2}$ & Percepção ambiental +TDHA & 9 & 3 \\
\hline $\mathbf{3}$ & Sala de aula + TDHA & 8 & 0 \\
\hline $\mathbf{4}$ & Percepção ambiental +Sala de aula & 97 & 2 \\
\hline $\mathbf{5}$ & Ambiente de estudo + deficiência cognitiva & 180 & 0 \\
\hline $\mathbf{6}$ & Sala de aula + Deficiência cognitiva & 31 & 0 \\
\hline $\mathbf{7}$ & Ergonomia do ambiente + Ambiente de estudo & 74 & 4 \\
\hline $\mathbf{8}$ & Ambiente de Estudo + TDAH & 26 & $\mathbf{1 2}$ \\
\hline & & 442 & 26 \\
\hline
\end{tabular}

Fonte: As autoras, 2017.

Diante de um resultado de 442 artigos encontrados, após exclusão a partir do título, filtro dos últimos 5 anos e revisados por pares, apenas 12 poderão contribuir para a pesquisa, por ter relação direta com o tema. Muitos o tratavam sob a ótica pedagógica, médica, softwares e educação online. Nessa plataforma, as combinações que mais trouxeram resultados em número foi Percepção ambiental com Sala de aula e Ambiente de estudo com deficiência cognitiva, porém as combinações que mais trouxeram artigos relacionados com o tema foram: ambiente de estudo com TDAH e sala de aula com TDAH, dando um número de 10 artigos relevantes, dos 12 encontrados. Na tabela 4 são listados os artigos selecionados da Capes através das combinações em Português, na coluna observações foi justificado a escolha de cada artigo para contribuir com a pesquisa, relacionando resumidamente com 0 tema.

Tabela 4: Artigos relevantes encontrados através das combinações em português na CAPES.

\begin{tabular}{|c|l|l|}
\hline CAPES & \multicolumn{1}{|c|}{ TíTULOS } & \multicolumn{1}{|c|}{ OBSERVAÇÕES } \\
\hline 1 & Design e ergonomia: aspectos tecnológicos & $\begin{array}{l}\text { Possui 2 capítulo relacionados com o } \\
\text { tema: } \\
- \text { Estudo ergonômico ambiental de } \\
\text { escolas das cidades de Bauru e Lençóis } \\
\text { Paulista }\end{array}$ \\
\hline
\end{tabular}




\begin{tabular}{|c|c|c|}
\hline & & $\begin{array}{l}\text { - Condições ambientais em escolas } \\
\text { municipais de ensino infantil da cidade } \\
\text { de Marília (São Paulo): estudo de caso }\end{array}$ \\
\hline 2 & $\begin{array}{l}\text { Transtorno de Déficit de } \begin{array}{l}\text { Atenção/ } \\
\text { Hiperatividade: diagnóstico da prática } \\
\text { pedagógica }\end{array} \\
\end{array}$ & $\begin{array}{l}\text { Definição de TDAH. } \\
\text { Fala sobre a relação das escolas com o } \\
\text { TDAH. }\end{array}$ \\
\hline 3 & $\begin{array}{l}\text { Aprendizagem e desenvolvimento humano: } \\
\text { avaliações e intervenções }\end{array}$ & $\begin{array}{l}\text { Possui } 1 \text { capítulo relacionado com o } \\
\text { tema: } \\
\text { - Concepções dos professores do } \\
\text { ensino fundamental sobre TDAH. } \\
\text { (Contém dados sobre o TDAH) }\end{array}$ \\
\hline 4 & $\begin{array}{l}\text { Sistema de economia de fichas associado ao } \\
\text { custo da resposta aplicado aos } \\
\text { comportamentos da sala de aula de } \\
\text { adolescente com TDAH. }\end{array}$ & $\begin{array}{l}\text { Fala sobre a eficácia da intervenção } \\
\text { positiva do professor no processo de } \\
\text { aprendizagem do aluno com TDAH. }\end{array}$ \\
\hline 5 & $\begin{array}{l}\text { Competição social e status sociométrico em } \\
\text { escolares diagnosticados com transtorno de } \\
\text { Déficit de Atenção e Hiperatividade (TDAH). }\end{array}$ & $\begin{array}{l}\text { Estudo das relações entre auto } \\
\text { percepção da competição social em } \\
\text { crianças com TDAH para fortalecer a } \\
\text { interação social dessas crianças na sala } \\
\text { de aula. }\end{array}$ \\
\hline 6 & $\begin{array}{l}\text { Categorias de necessidades educacionais } \\
\text { especiais enquanto preditoras de déficits em } \\
\text { habilidades sociais na infância. }\end{array}$ & $\begin{array}{l}\text { Esta pesquisa verificou a força preditiva } \\
\text { de } 12 \text { categorias de necessidades } \\
\text { educacionais especiais sobre } \\
\text { repertorio de habilidades sociais de } \\
\text { crianças. Mais focado nas habilidades } \\
\text { sociais. }\end{array}$ \\
\hline 7 & $\begin{array}{l}\text { Evidências preliminares da efetividade do } \\
\text { treinamento cognitivo para melhorar a } \\
\text { inteligência de crianças escolares. }\end{array}$ & $\begin{array}{l}\text { Teste experimental cognitivo feito com } \\
\text { crianças com media de } 8 \text { anos. }\end{array}$ \\
\hline 8 & $\begin{array}{l}\text { Um programa de treinamento dos processos } \\
\text { da atenção para crianças. }\end{array}$ & $\begin{array}{l}\text { Tradução, adaptação e análise de } \\
\text { aplicabilidade clínica do Programa Pay } \\
\text { Attention! ao português do Brasil. }\end{array}$ \\
\hline 9 & $\begin{array}{l}\text { Transtorno de Déficit de Atenção/ } \\
\text { Hiperatividade: um olhar voltado para a } \\
\text { escola. }\end{array}$ & $\begin{array}{l}\text { Fala sobre a relação da escola com os } \\
\text { seus alunos com TDAH, evidenciando } \\
\text { qual o seu papel frente ao transtorno. }\end{array}$ \\
\hline 10 & $\begin{array}{l}\text { Construção de uma escala para avaliação do } \\
\text { planejamento cognitivo. }\end{array}$ & $\begin{array}{l}\text { Construção de uma escala de auto } \\
\text { relato para avaliar o planejamento } \\
\text { cognitivo em adultos. }\end{array}$ \\
\hline
\end{tabular}

Fonte: As autoras, 2017.

Foram encontrados 10 artigos relevantes pelo portal Capes. A mesma metodologia foi aplicada ao portal Scielo, com as palavras em português. A tabela 5 busca explanar de forma didática e prática essas pesquisas: 
Tabela 5: Quantitativo de resultados encontrados pelas combinações em português na SCIELO.

\begin{tabular}{|c|c|c|c|}
\hline SCIELO & COMBINAÇÕES & $\begin{array}{c}\text { RESULTADOS DA } \\
\text { BUSCA }\end{array}$ & $\begin{array}{c}\text { RESULTADOS } \\
\text { RELEVANTES }\end{array}$ \\
\hline $\mathbf{1}$ & Ergonomia do ambiente + Sala de aula & 0 & 0 \\
\hline $\mathbf{2}$ & Percepção ambiental +TDHA & 0 & 0 \\
\hline $\mathbf{3}$ & Sala de aula + TDHA & 3 & 2 \\
\hline $\mathbf{4}$ & Percepção ambiental + Sala de aula & 3 & 0 \\
\hline $\mathbf{5}$ & Ambiente de estudo + Deficiência cognitiva & 1 & 1 \\
\hline $\mathbf{6}$ & Sala de aula + Deficiência cognitiva & 1 & 0 \\
\hline $\mathbf{7}$ & Ergonomia do ambiente + Ambiente de estudo & 32 & 0 \\
\hline $\mathbf{8}$ & Ambiente de Estudo + TDAH & 0 & $\mathbf{4}$ \\
\hline & & $\mathbf{3 4}$ & \\
\hline
\end{tabular}

Fonte: As autoras, 2017.

De acordo com tabela 5 os resultados de busca nesta plataforma foram bem inferiores ao da plataforma Capes, gerando apenas 34, nos quais apenas 4 tem relação direta com tema. As combinações que mais geraram resultados em número foram ergonomia do ambiente com ambiente de estudo, porém, nenhum deles alcançou um grau de relevância satisfatório para o tema escolhido a ser abordado no artigo. Além disso, a combinação que mais encontrou artigo relevante foi sala de aula com TDAH. A tabela 6 consiste em explanar os artigos úteis com sua justificativa de escolha através do título e leitura do resumo.

Tabela 6: Artigos relevantes encontrados através das combinações em português no SCIELO.

\begin{tabular}{|c|l|l|}
\hline SCIELO & \multicolumn{1}{|c|}{ TÍTULOS } & \multicolumn{1}{|c|}{ OBSERVAÇÕES } \\
\hline $\mathbf{1}$ & $\begin{array}{l}\text { Competencia social y status sociométrico } \\
\text { escolar en niños y niñas con TDAH. }\end{array}$ & $\begin{array}{l}\text { Aborda a questão da interação social } \\
\text { dos alunos com TDAH. }\end{array}$ \\
\hline $\mathbf{2}$ & $\begin{array}{l}\text { Transtorno de conduta/TDAH e aprendizagem } \\
\text { da Matemática: um estudo de caso. }\end{array}$ & $\begin{array}{l}\text { Apesar de falar da prática pedagógica, } \\
\text { ajuda a entender as necessidades de } \\
\text { aprendizagem do aluno com TDAH } \\
\text { para transpor no ambiente da sala de } \\
\text { aula. Destaque para o ambiente } \\
\text { multifuncional. }\end{array}$ \\
\hline $\mathbf{3}$ & $\begin{array}{l}\text { Estratégias pedagógicas empregadas por } \\
\text { professores de educação especial aos seus } \\
\text { alunos com deficiência intelectual severa: um } \\
\text { estudo descritivo da prática docente. }\end{array}$ & $\begin{array}{l}\text { Foco na educação de indivíduos com } \\
\text { deficiência intelectual severa. Também } \\
\text { fala da importância do professor como } \\
\text { mediador no ambiente da sala de aula. }\end{array}$ \\
\hline $\mathbf{4}$ & $\begin{array}{l}\text { Proyecto Mosqueteros: una propuesta de } \\
\text { intervención en el aula para favorecer los } \\
\text { procesos de inclusión escolar. }\end{array}$ & $\begin{array}{l}\text { O presente trabalho procurou avaliar } \\
\text { se o projeto Mosqueteiros, desenhado } \\
\text { para gerar ambientes sociais } \\
\text { adequados para sua inclusão escolar, } \\
\text { poderia aumentar a interação entre }\end{array}$ \\
\hline
\end{tabular}


Fonte: As autoras, 2017.

Foi realizado o mesmo procedimento, nas mesmas plataformas de pesquisa, anteriormente apresentado, com as correspondentes palavras chaves na língua inglesa. A tabela 7 e 8 mostra o quantitativo de resultados encontrados através das combinações em inglês no portal CAPES e os títulos com suas observações e justificativas de sua seleção.

Tabela 7: Quantitativo de resultados encontrados pelas combinações em inglês na CAPES.

\begin{tabular}{|c|c|c|c|}
\hline CAPES & COMBINAÇÕES & $\begin{array}{c}\text { RESULTADOS } \\
\text { DA BUSCA }\end{array}$ & $\begin{array}{c}\text { RESULTADOS } \\
\text { RELEVANTES }\end{array}$ \\
\hline $\mathbf{1}$ & Build environment ergonomics + Classroom & 562 & 1 \\
\hline $\mathbf{2}$ & Environmental perception +ADHD & 3 & 0 \\
\hline $\mathbf{3}$ & Classroom + ADHD & 7.732 & 5 \\
\hline $\mathbf{4}$ & Environmental perception + Classroom & 65 & 1 \\
\hline $\mathbf{5}$ & Place of study + Cognitive deficiency & 24.130 & 0 \\
\hline $\mathbf{6}$ & Classroom + Cognitive deficiency & 3.672 & 2 \\
\hline $\mathbf{7}$ & Build environment ergonomics + Place of study & 4.058 & 0 \\
\hline $\mathbf{8}$ & Place of study + ADHD & 16.944 & 3 \\
\hline & & $\mathbf{5 7 . 1 6 6}$ & $\mathbf{1 2}$ \\
\hline
\end{tabular}

Fonte: As autoras, 2017.

Tabela 8: Artigos relevantes encontrados através das combinações em inglês na CAPES.

\begin{tabular}{|c|l|l|}
\hline CAPES & \multicolumn{1}{|c|}{ TíTULOS } & \multicolumn{1}{|c|}{ OBSERVAÇÕES } \\
\hline $\mathbf{1}$ & $\begin{array}{l}\text { Enabling outcomes for students with } \\
\text { developmental disabilities through } \\
\text { collaborative consultation.(Report) }\end{array}$ & TEXTO NÃO DISPONIVEL \\
\hline $\mathbf{2}$ & $\begin{array}{l}\text { Variability in classroom social } \\
\text { communication: performance of children with } \\
\text { fetal alcohol spectrum disorders and typically } \\
\text { developing peers.(Report) }\end{array}$ & $\begin{array}{l}\text { Fala sobre a usabilidade de um celular } \\
\text { como instrumento de aprendizagem, e } \\
\text { como forma de prender a atenção dos } \\
\text { alunos em sala de aula. }\end{array}$ \\
\hline $\mathbf{3}$ & $\begin{array}{l}\text { Improving self-monitoring and self-regulation: } \\
\text { From cognitive psychology to the classroom }\end{array}$ & $\begin{array}{l}\text { Psicologia cognitiva e contribuição } \\
\text { para melhorias na intervenção } \\
\text { educacional. }\end{array}$ \\
\hline
\end{tabular}




\begin{tabular}{|c|c|c|}
\hline 4 & $\begin{array}{l}\text { The impact of instructional context on } \\
\text { classroom on-task behavior: A matched } \\
\text { comparison of children with ADHD and non- } \\
\text { ADHD classmates }\end{array}$ & $\begin{array}{l}\text { Comparação entre crianças com } \\
\text { TDHA e não TDHA em sala de aula }\end{array}$ \\
\hline 5 & $\begin{array}{l}\text { The Patient's Perspective on the Link } \\
\text { Between ADHD and Substance Use }\end{array}$ & $\begin{array}{l}\text { Ensaio clinico randomizado com } 102 \\
\text { crianças com TDHA (Medicadas com } \\
\text { não). }\end{array}$ \\
\hline 6 & $\begin{array}{l}\text { Computer use in educational activities by } \\
\text { students with ADHD }\end{array}$ & $\begin{array}{l}\text { Uso de computadores nas atividades } \\
\text { em aula para estudantes com TDHA }\end{array}$ \\
\hline 7 & $\begin{array}{l}\text { The impact of classroom settings on } \\
\text { students' seat-selection and academic } \\
\text { performance }\end{array}$ & $\begin{array}{l}\text { Configurações da sala de aula e } \\
\text { desempenho acadêmico. }\end{array}$ \\
\hline 8 & $\begin{array}{l}\text { Classroom Changes in ADHD Symptoms } \\
\text { Following Clinic-Based Behavior Therapy }\end{array}$ & $\begin{array}{l}\text { Estudo sobre o comportamento da } \\
\text { criança com TDAH na sala de aula }\end{array}$ \\
\hline 9 & $\begin{array}{l}\text { The impact of instructional context } \\
\text { on classroom on-task behavior: A matched } \\
\text { comparison of children with ADHD and non- } \\
\text { ADHD classmates. }\end{array}$ & $\begin{array}{l}\text { Reação de criança flamengas com } \\
\text { TDAH na sala de aula, que tiveram } \\
\text { seus medicamentos cortados. (Foco } \\
\text { na área médica e pedagógica). }\end{array}$ \\
\hline 10 & $\begin{array}{l}\text { Designing an iPad App to Monitor and } \\
\text { Improve ClassroomBehavior for Children } \\
\text { with ADHD: iSelfControl Feasibility and Pilot } \\
\text { Studies }\end{array}$ & $\begin{array}{l}\text { Utiliade de um app no comportamento } \\
\text { de crianças com TDAH em sala de } \\
\text { aula. }\end{array}$ \\
\hline 11 & $\begin{array}{l}\text { Self-regulation strategies support children } \\
\text { with ADHD to overcome symptom-related } \\
\text { behavior in the classroom }\end{array}$ & $\begin{array}{l}\text { Três estudos de caso de intervenções } \\
\text { em salas de aula. }\end{array}$ \\
\hline 12 & $\begin{array}{l}\text { Younger Children in the Classroom Likely } \\
\text { Overdiagnosed With ADHD }\end{array}$ & TEXTO NÃO DISPONIVEL \\
\hline
\end{tabular}

Fonte: As autoras, 2017.

O número de resultados úteis se assemelhou muito à pesquisa realizada em português, com um total de 12 resultados. A combinação que mais gerou resultados foi Classroom com $A D D H$.

Devido à escassez de resultados encontrados para o tema chave, considerando a pesquisa ter se limitado a apenas dois sistemas de buscas, é possível avaliar que o tema ainda é pouco explorado e a teoria neste campo ainda está se formando. Utilizando já a string "Build environment ergonomics" nas pesquisas em inglês da Scielo, a tabela 9 e 10 explana os resultados gerados pela plataforma de busca, seu quantitativo e justificativa, respectivamente.

Tabela 9: Quantitativo de resultados encontrados pelas combinações em inglês na SCIELO.

\begin{tabular}{|c|c|c|c|}
\hline SCIELO & COMBINAÇÕES & $\begin{array}{c}\text { RESULTADOS } \\
\text { DA BUSCA }\end{array}$ & $\begin{array}{l}\text { RESULTADOS } \\
\text { RELEVANTES }\end{array}$ \\
\hline
\end{tabular}




\begin{tabular}{|c|c|c|c|}
\hline $\mathbf{1}$ & Build environment ergonomics + Classroom & 0 & 0 \\
\hline $\mathbf{2}$ & Ergonomics + Classroom & 6 & 0 \\
\hline $\mathbf{3}$ & Environmental perception +ADHD & 0 & 0 \\
\hline $\mathbf{4}$ & Classroom + ADHD & 10 & 4 \\
\hline $\mathbf{5}$ & Environmental perception + Classroom & 2 & 0 \\
\hline $\mathbf{6}$ & Place of study + Cognitive deficiency & 0 & 0 \\
\hline $\mathbf{7}$ & Classroom + Cognitive deficiency & 1 & 1 \\
\hline $\mathbf{8}$ & Build environment ergonomics + Place of study & 0 & 0 \\
\hline $\mathbf{9}$ & Place of study + ADHD & 3 & 0 \\
\hline & & $\mathbf{2 2}$ & $\mathbf{5}$ \\
\hline
\end{tabular}

Fonte: As autoras, 2017.

Tabela 10: Artigos relevantes encontrados através das combinações em inglês na SCIELO.

\begin{tabular}{|c|c|c|}
\hline SCIELO & TÍTULOS & OBSERVAÇÕES \\
\hline 1 & $\begin{array}{l}\text { A medicalização da educação: implicações } \\
\text { para a constituição do sujeito/aprendiz }\end{array}$ & $\begin{array}{l}\text { Fala sobre as vertentes do TDAH: } \\
\text { organicista e sócio-histórica. }\end{array}$ \\
\hline 2 & $\begin{array}{l}\text { Competencia social y status sociométrico } \\
\text { escolar en niños y niñas con TDAH }\end{array}$ & $\begin{array}{l}\text { ARTIGO REPETIDO, N.5. DA CAPES } \\
\text { (TABELA 4) }\end{array}$ \\
\hline 3 & $\begin{array}{l}\text { Habilidades motoras y de procedimiento que } \\
\text { interfieren en la vida académica habitual de } \\
\text { un grupo de estudiantes con signos y } \\
\text { síntomas de TDAH }\end{array}$ & $\begin{array}{l}\text { Fala da importância de uma } \\
\text { intervenção especializada para } \\
\text { conduzir a educação de um estudante } \\
\text { com TDAH. }\end{array}$ \\
\hline 4 & $\begin{array}{l}\text { Methods for Assessing Social Validity of } \\
\text { Behavioral Intervention Plans for Children } \\
\text { with Attention Deficit }\end{array}$ & $\begin{array}{l}\text { Analisa o quão é valido são os planos } \\
\text { de intervenção de comportamento na } \\
\text { vida dos alunos com TDAH. }\end{array}$ \\
\hline 5 & $\begin{array}{l}\text { Potenciais dificuldades e facilidades na } \\
\text { educação de alunos com deficiência } \\
\text { intelectual }\end{array}$ & $\begin{array}{l}\text { Define possibilidades da prática } \\
\text { pedagógica no contexto escolar para } \\
\text { alunos com deficiência intelectual. }\end{array}$ \\
\hline
\end{tabular}

Fonte: As autoras, 2017.

Assim como no Periódico da CAPES, a combinação que gerou mais resultados relevantes sobre o tema foi Classroom com $A D H D$, gerando 4 resultados de 5 totais. Os resultados de busca da SCIELO foram melhores em português, mas nas duas plataformas a troca de palavras chave para inglês fez-se necessário para analisar a maior quantidade de artigos possíveis. 


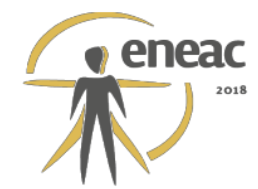

\section{ANÁLISE DA RSL}

Conclui-se a revisão sistemática da literatura com uma visão geral do que foi pesquisado, explanado na tabela 11. Nela é possível observar o quantitativo dos resultados encontrados nos portais CAPES e SCIELO, pesquisado no idioma inglês e português e mostra quanto o quanto cada plataforma contribuiu para a pesquisa com a coluna de resultados relevantes.

Tabela 11: Quantitativo de resultados encontrados plataformas de pesquisa.

\begin{tabular}{|c|c|c|}
\hline \multicolumn{2}{|c|}{ RESULTADOS GERAIS OBTIDOS POR CADA PLATAFORMA } \\
\hline PLATAFORMA & RESULTADOS DA BUSCA & RESULTADOS RELEVANTES \\
\hline Capes & 57.608 & 22 \\
\hline Scielo & 34 & 8 \\
\hline
\end{tabular}

Fonte: As autoras, 2017.

Proporcionalmente aos resultados de busca, apenas $0,03 \%$ dos resultados encontrados na CAPES foram relevantes, enquanto na Scielo, $26 \%$ dos encontrados contribuíram para a pesquisa. No gráfico 1, são abordados os países de maior pesquisa sobre esse tema, 0 Brasil é o local onde mais se pesquisa sobre a ergonomia do ambiente construído da sala de aula e sobre a percepção dos alunos com TDAH no seu ambiente de estudo, por isso, os resultados da pesquisa em português foi tão enriquecedor.

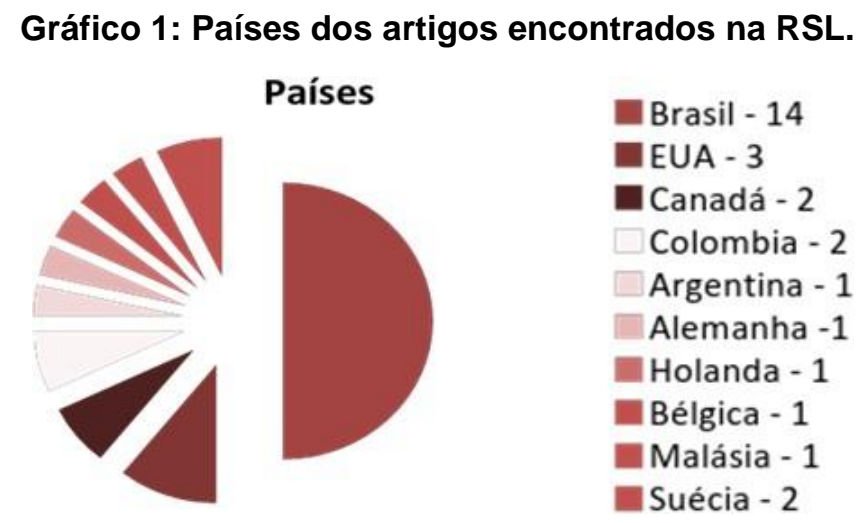

Fonte: As autoras, 2017.

Ainda existe uma lacuna a ser estudada sobre a percepção dos alunos com TDAH em sala de aula e ergonomia da sala propriamente dita. Não obtendo um grande número de fontes para realizar um referencial teórico mais aprofundado como era esperado.

Apesar do pouco número de artigos que contribuíssem para o estudo, mesmo após pesquisa em português e em inglês, os dados coletados foram bastante consistentes para compor um referencial inicial para embasar o tema em questão. 


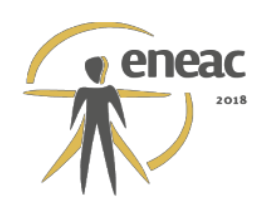

\section{CONCLUSÃO}

Diante dos resultados da RSL e análise de seus dados é possível concluir que nos motores de buscas utilizados, Capes e Scielo, pouco ainda é publicado sobre o tema da ergonomia da sala de aula, e menos ainda focando no aluno com TDAH.

Em contrapartida há um expressivo quantitativo de publicações sobre o TDAH. Segundo Bonadio e Mori (2013), a temática do TDAH não é recente no meio acadêmico, muitas pesquisas foram e estão sendo realizadas, com enfoque maior nos aspectos orgânicos, pautados principalmente no uso de medicamentos. O TDAH se legitima normalmente aos diagnósticos médicos e psiquiátricos, porém, é um produto de circunstâncias históricas e sociais, internas e externas ao campo médico, que ocupa um espaço configurado pelo traço biológico, cerebral, epidemiológico, mas também moral, social e existencial. (DOMINGUES; ZANCANELLA; BASEGGIO, 2013).

Partindo do princípio de que o social e o moral têm seu peso e influencia o comportamento das crianças com TDAH, temos então, o ambiente escolar como parte essencial para a formação desses indivíduos.

Entendendo a especificidade de educação de grupo mais diferenciado é que esta RSL sugere um pensamento de trabalho mais inclusivo e adaptável, visto a escassez de exemplos de ambientes ergonômicos aplicados a alunos com TDAH.

Segundo Domingues, Zancanella e Baseggio (2013), ao relacionar a problemática do TDAH com a escola, deve-se considerar o olhar atento necessário ao profissional de educação que é indispensável para auxiliar seus educandos em suas dificuldades e, para tanto, precisa compreender também o contexto de vida da criança.

Quanto ao ambiente escolar, estes autores dizem que este deve complementar o ambiente familiar do educando, ser agradável e gerador de afeto. Eles ainda citam o conceito da teoria de Winnicott, a qual diz que o ambiente deve ser um facilitador do processo de aprendizagem da criança em desenvolvimento; da mesma forma, o professor/escola deve proporcionar um ambiente que estimule e, ao mesmo tempo, acolha a criança no seu tempo de aprender.

Haghighi e Jusan (2015) dizem que as barreiras físicas (acústica, temperatura, luminosidade) são elementos que interferem diretamente na relação de bem estar entre o homem e o ambiente, independente de idade e referência cultural. Com isso temos que os condicionantes físicos são importantes para o bem estar de qualquer um dos usuários de sala de aula, seja esse portador de TDAH ou não.

Sob tais premissas torna-se necessário e interessante a realização de pesquisas que foquem na percepção ambiental do aluno com TDAH e consiga avaliar seu comportamento no ambiente de atividade, e que direcione o profissional de arquitetura/design/engenharia como manejar e aplicar esses fatores ergonômicos.

Compreendendo que a influência da ergonomia da sala de aula nas questões do aprendizado das crianças é algo tangível, são necessárias pesquisas mais aprofundadas, sobre dados específicos e até práticos da ergonomia do ambiente construído da sala de aula para alunos com TDAH.

\section{REFERÊNCIAS BIBLIOGRÁFICAS}

ATALLAH, N.A.; CASTRO A.A. Revisões sistemáticas da literatura e metanálise: a melhor forma de evidência para tomada de decisão em saúde e a maneira mais rápida de atualização terapêutica. Diagnóstico \& Tratamento.1997. P.20-22. 
Atenção/Hiperatividade: diagnóstico e prática pedagógica. Maringá: Eduem, 2013.

DOMINGUES, Leane; ZANCANELLA, Sabrina; BASEGGIO, Denice Bortolin. Transtorno de Déficit de Atenção/Hiperatividade: um olhar voltado para a escola. Barbarói, Santa Cruz do Sul, n.39, jul./dez. 2013. p.149-163

HAGHIGHI, Mohammad Moslemi; JUSAN, Mahmud Bin Mohd. The impact of classroom settings on students' seat-selection and academic performance. [Editorial]. Indoor and Built Environment., Vol. 24, n. 2, p. 280-288, set. 2015.

Portal Capes. Disponível em: <http://www.periodicos.capes.gov.br/>. Acesso em: 18 abr. 2017.

Portal Scielo. Disponível em: <http://www.scielo.org.co/>. Acesso em: 23 maio. 2017. 